\title{
Management of multiple sclerosis patients in central European countries: current needs and potential solutions
}

\author{
Thomas Berger, Monika Adamczyk-Sowa, Tünde Csépány, Franz Fazekas, \\ Tanja Hojs Fabjan, Dana Horáková, Zsolt Illes, Eleonóra Klimová, Fritz Leutmezer, \\ Konrad Rejdak, Csilla Rozsa, Saša Šega Jazbec, Jarmila Szilasiová, Peter Turčáni, \\ Marta Vachová, László Vécsei and Eva Havrdová
}

\begin{abstract}
Multiple sclerosis (MS) experts in Europe are facing rapidly rising demands of excellence due to the increasing complexity of MS therapy and management. A central European expert board of MS experts met to identify needs and obstacles with respect to raising quality of MS care in central and Eastern European countries. There are substantial variations across countries regarding delivery of care and its cost structure, as well as access to treatment. To date, Eastern European countries are often less able to afford reimbursement of immunomodulatory agents than Western countries. Overall, approximately $40 \%$ of working-age patients are not working due to MS. Costs rise steeply with increasing disability; indirect costs constitute the bulk of the financial burden in patients with severe MS. Magnetic resonance imaging (MRI) assessment is meanwhile obligatory as the diagnostic interface in the management of MS patients. Recommended measures directed at improving quality of care include the collection of patient data in registries, enhanced education of healthcare professionals, implementation of national strategies aiming at reducing regional variation, optimization of approval processes, and removal of administrative barriers. Local partnerships with authorities such as those that represent the interests of employees can contribute to leverage the importance of epidemiological data. The need for education extends to (neuro)radiologists who are responsible for reporting MRI findings in expert quality. Dissemination of the Magnetic Resonance Imaging in MS (MAGNIMS) protocol would be an important step in this context. Also, clinical freedom of choice is rated as essential. Physicians should have access to a range of treatment options due to the complexity of disease. Guidelines such as the upcoming EAN-ECTRIMS clinical practice guideline also aim at providing a basis for argumentation in negotiations with national health authorities.
\end{abstract}

Keywords: access to treatment, burden of disease, MS registry, multiple sclerosis, quality of care

Received: 8 June 2017; revised manuscript accepted: 4 September 2017.

\section{Introduction}

The central European MS Expert Board was founded in 2007 with the aim of improving the management and (availability of) care of multiple sclerosis (MS) patients in the central European area. The original member countries include Austria, the Czech Republic, Hungary, Poland, Slovakia, and Slovenia. Renowned MS experts from these countries have been convening for periodical meetings to share practical aspects including local treatment algorithms, educational requirements, data gaps, and support for local argumentation/ negotiation gaps with health authorities.

It was generally agreed that there is a need to improve care of MS patients in the light of increasing complexity of MS therapy and heightened demands in excellence at MS centers. Identifying
Ther Adv Neurol Disord

2018, Vol. 11: 1-12

DOI: 10.1177/

1756286418759189

(c) The Author(s), 2018.

Reprints and permissions: http://www.sagepub.co.uk/ journalsPermissions.nav

\section{Correspondence to: \\ Thomas Berger Clinical Department of Neurology, Medical University of Innsbruck, Anichstrasse 35, A-6020 Innsbruck, Austria thomas.bergerฉi-med. ac.at \\ Eva Havrdová Dana Horáková Department of Neurology and Center of Clinical Neuroscience, First Faculty of Medicine, Charles University and General University Hospital, Prague, Czech Republic}

Monika Adamczyk-Sowa Department of Neurology in Zabrze, Medical University of Silesia, Katowice, Poland

Tünde Csépány Department of Neurology, Faculty of Medicine. University of Debrecen, Debrecen, Hungary

Tanja Hojs Fabjan Department of Neurology, University Medical Centre Maribor, Maribor, Slovenia Franz Fazekas

Department of Neurology, Medical University of Graz,

Graz, Austria

Zsolt Illes

Department of Neurology, University of Southern Denmark, Odense, Denmark

Eleonóra Klimová Department of Neurology, University of Prešov and Teaching Hospital of JA Reiman, Prešov, Slovakia

Fritz Leutmezer Department of Neurology, Medical University of Vienna, Vienna, Austria Konrad Rejdak Department of Neurology, Medical University of Lublin, Lublin, Poland Csilla Rozsa 
Department of

Neurology, Jahn

Ferenc Dél-pesti

Hospital, Budapest,

Hungary

Saša Šega Jazbec

Department of

Neurology, University

of Ljubljana,

Ljubljana, Slovenia

Jarmila Szilasiová

Department of

Neurology, Pavol

Jozef Šafárik

University and

University Hospital

L Pasteur, Košice,

Slovakia

Peter Turčáni

Department

of Neurology,

Comenius University

Bratislava, Slovakia

Marta Vachová

MS Centrum Teplice,

Teplice, Czech

Republic

László Vécsei

Department of

Neurology and MTA-

SZTE Neuroscience

Research Group,

University of Szeged,

Szeged, Hungary
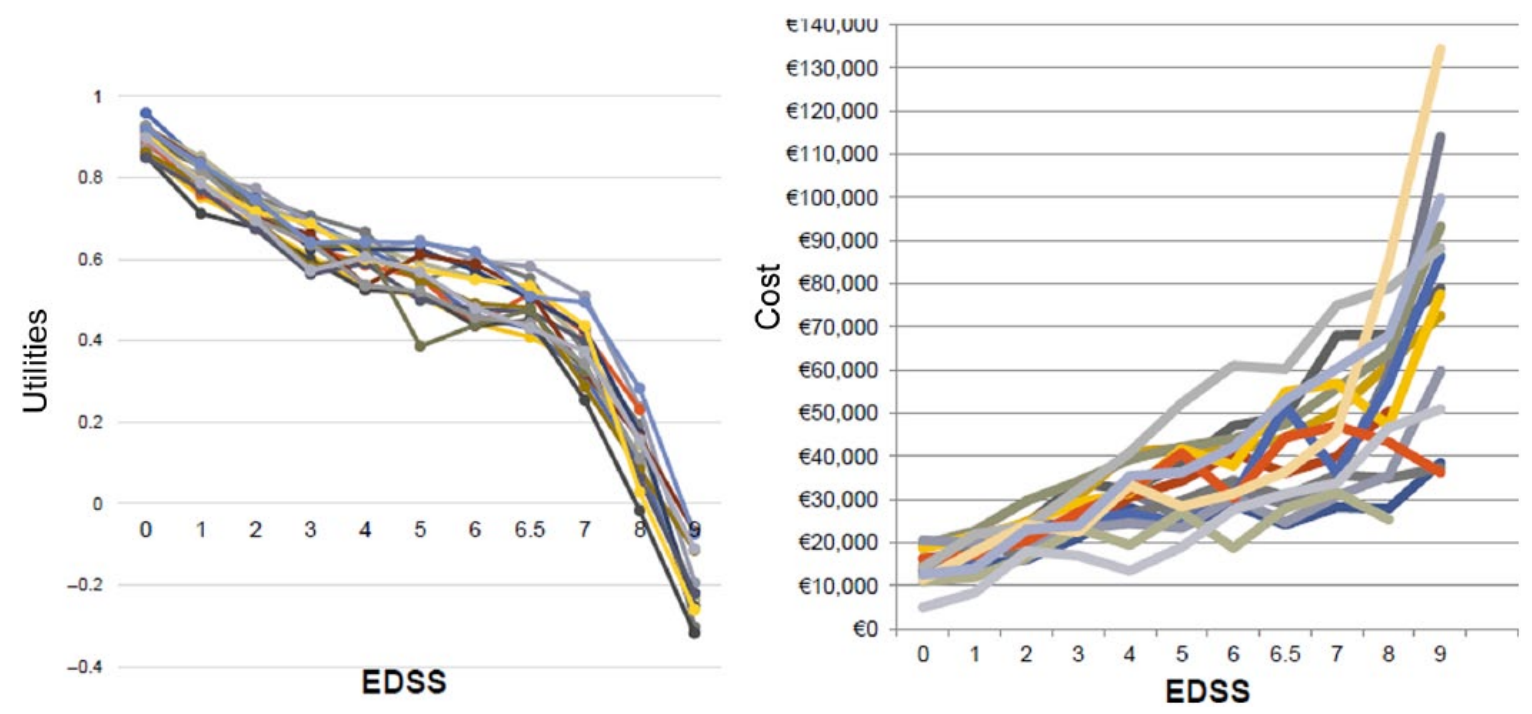

Figure 1. Effect of disease progression in multiple sclerosis patients on quality of life (left) and financial burden (right) in 16 European countries.

Source: Kobelt et al. ${ }^{1}$

EDSS, Expanded Disability Status Scale.

local needs, even if potential measures appear visionary at the time of their discussion, has become an important part of the board's work. The expert board is being perceived as a solutionoriented forum guided by pragmatism and transparency.

This paper summarizes the content discussed at the most recent board meeting held on January 21,2017 , in Vienna, Austria. Lectures and debates focused on gaps in daily MS management in the respective countries and prioritized problem-solving approaches.

\section{Burden of disease in Europe}

Similarities and differences with respect to the burden of MS across Europe have been assessed by the observational, cross-sectional, retrospective MS Cost Of Illness (MS COI) Study that contains data from 16,808 patients in 16 countries (Austria, Belgium, Czech Republic, Denmark, France, Germany, Hungary, Italy, Netherlands, Poland, Portugal, Russia, Spain, Sweden, Switzerland, United Kingdom). ${ }^{1}$ MS COI is a patient survey based on paper and online questionnaires that were distributed by 18 patient advocacy groups. It thus provides a snapshot of the experience of the responding patients, rather than representing a prevalence sample. The findings can therefore be expected to bear a certain selection bias; however, generally speaking, they are in keeping with other large datasets of a similar nature. ${ }^{2,3}$ Individual papers on the MS COI results will be published for each country this year.

The survey population shows the full range of disease severity according to the Expanded Disability Status Scale (EDSS). Mean EDSS scores for the individual countries ranged from 2.9 (Russia) to 5.5 (United Kingdom). However, comparisons across countries are difficult due to inherent variations with regard to healthcare systems, medical traditions, purchasing power and other parameters. Also, as this is a cross-sectional study, causal inference is not possible.

A consistent finding across Europe was the steep decrease in quality of life as EDSS increases (Figure 1), which illustrates the need to avert disease progression. At the same time, the total costs increased dramatically. According to the analysis of cost structure, expenses for disease-modifying drugs (DMDs) amounted to $27 \%$ in the total study population (Figure 2). However, the largest component constituted indirect costs relating to factors such as production losses and caregiver burden. Due to differences in mechanisms of care delivery, types of patients and levels of severity, cost structure varied considerably across countries. This also applied to types of hospitalization (percentages of inpatient stays, day admissions, 

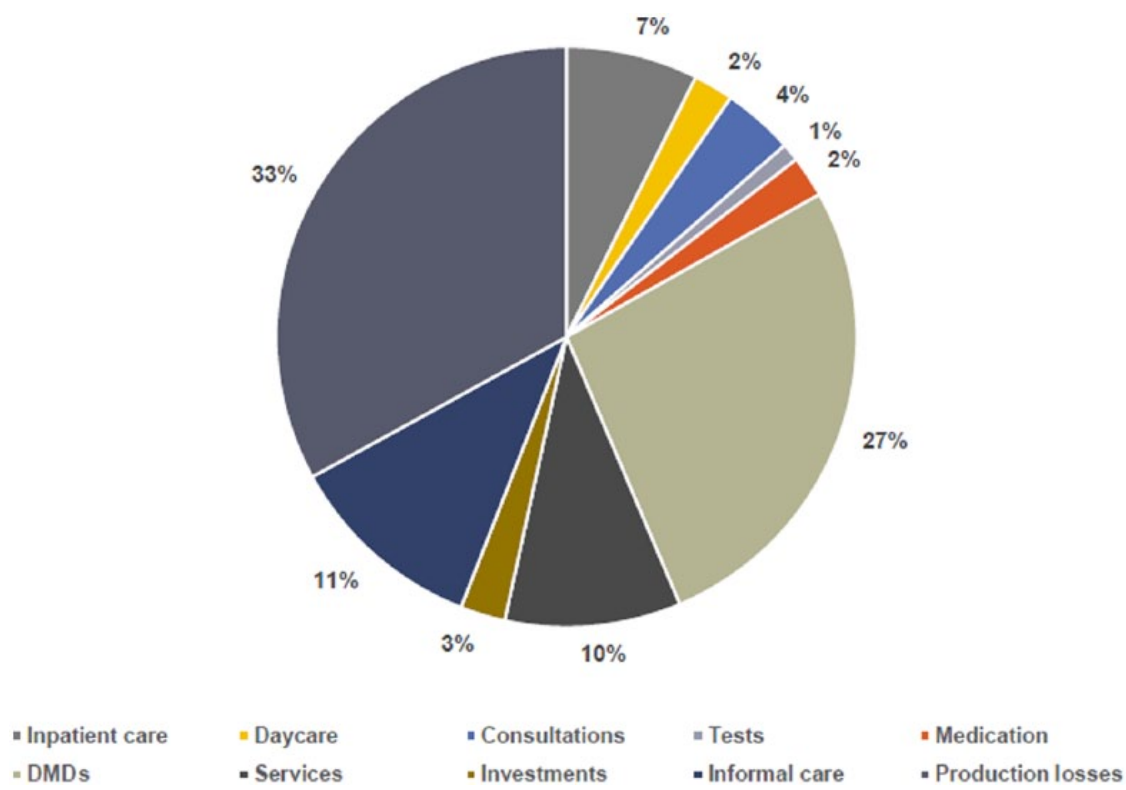

Figure 2. Cost structure in the Multiple Sclerosis Cost of Illness (MS COI) study in the total study population ( $n=16,808)$.

Kobelt et al. ${ }^{1}$

DMDs, disease-modifying drugs.

use of rehabilitation and nursing home services) and types of healthcare professionals that MS patients tend to consult. Here, the proportions of consultations with neurologists, MS nurses, general practitioners and physiotherapists differed conspicuously across countries. On average, $22.5 \%$ of patients reported MRI brain examinations within the previous 3 months.

The mean cost of relapses occurring during a 3-month reporting period was estimated at EUR 2188 (adjusted for purchasing power); wide variations were seen in these costs, with country means ranging from EUR 632 to EUR 4569. Approximately $40 \%$ of the total working-age population with MS were not working because of their disease at the time of the survey. This is a substantial proportion. Therefore, keeping the patients in the workforce and maximizing their ability to contribute productively to the economy should be an important treatment goal. Moreover, this aspect of the burden of MS is an essential factor to take into consideration when demonstrating the cost effectiveness of disease-modifying therapies.

Although pertinent data delineating the spectrum of MS treatment in European countries have been generated through this survey, it can be expected that national health authorities show little inclination to consider them in their daily proceedings, as their interest in diminishing indirect cost savings is generally limited. This might even be true for health insurances that only cover expenses for medical conditions and accidents, but not for retirement, which means that early retirement of an MS patient relieves them of their duties towards this patient. Therefore, there is agreement that it is advisable to form local partnerships with other authorities such as The Chamber of Labour in Austria that represents the interests of employees, with the aim of leveraging the importance of these epidemiological data. As a rule, providing local authorities with data on the burden of disease is preferable to waiting for them to present the MS community with data of their own.

\section{Access to treatment}

MS patients in all countries should have fair and equal access to the right treatments at the right time. However, this is not the case due to a number of reasons (Table 1). In 2013, 69\% of MS patients in Germany received DMDs, whereas in Poland, this only applied to $13 \% .^{4}$ Moreover, the percentages of patients being treated with innovative drugs for (highly) active MS was considerably higher in countries such as Norway, Sweden and Denmark than in Slovenia, Poland, or Romania. 
Table 1. Determinants of access to multiple sclerosis treatment.

+ Costs and the affordability of MS drugs

+ Differences in the reimbursement process and patient eligibility for treatment

+ Differences in diagnosis and clinical management of MS

+ Regional differences inside the same country

+ Use of patient registries or databases

MS, multiple sclerosis.

Table 2 lists the current availability and reimbursement status of various DMDs in Austria, the Czech Republic, Hungary, Poland, Slovenia, and Slovakia. Any limitations and restrictions are described.

These differences are mainly a consequence of the DMD-related impact on health budgets against the backdrop of disparities in wealth across European countries. Lower-income countries usually pay lower prices for drugs. In Romania, Slovenia, Poland and the Czech Republic, prices have decreased between 2008 and 2013. However, according to the affordability index, Eastern countries are still less able to afford immunomodulatory agents, even though the index has improved as well. The improvement of the affordability index can partly be ascribed to increased expenses on health care in Eastern countries over time.

Most of the established treatments for mild/moderate relapsing MS have become available in Eastern European countries. However, certain reimbursement restrictions, such as limitations of the number of treated patients or limitations of duration of treatment, still occur. There is a correlation between the level of access and the healthcare infrastructure. For instance, Slovenia has a shortage of neurologists, which means that waiting times for neurological examinations for MS patients presently amount to almost 2 years. The availability of MS centers and MS nurses is also relevant in this context, as are differences in local treatment recommendations/guidelines.

Only $30-40 \%$ of patients respond well to first-line treatment, but drug escalation is a major hurdle in less well-heeled countries. Physicians actually tend to be hesitant about treatment escalation in general.

Regional variations can be much larger than differences between Western and Eastern countries.
They include disparities in prescription behavior and referral of patients to more experienced hospitals. This is exemplified by the situation in Poland, where a substantial number of MS centers offers treatment for patients with mild-tomoderate relapsing disease, while drugs for highly active disease are prescribed only at a limited range of centers.

Another determinant of access is the use of registries or databases. A systematic survey has identified 20 European MS registries..$^{5}$ The collection of patient data in databases can help to optimize the management and advance knowledge about MS treatment in the local MS community. Cooperation and communication between MS centers is a crucial aspect here.

Several measures can improve patient access to treatment, such as the implementation of national strategies with the aim of reducing regional variation and devoting more resources to MS (e.g. increasing the numbers of neurologists and MS nurses). Decreases in costs of treatment are most likely to occur with the introduction of generics and biosimilars; however, governmental negotiations with the pharmaceutical companies also play a role here. Approval processes should be optimized, and administrative barriers should be removed. The collection of patient data through MS registries can contribute to enhanced access, as well as application of up-to-date national guidelines for treatment.

\section{The upcoming European Committee for the Treatment and Research in Multiple Sclerosis-European Academy of Neurology clinical practice guideline}

For authorities and physicians alike, the availability of guidelines on the management of MS that can be perceived as a European consensus is essential. The first European clinical practice guidelines on pharmacological management of MS will be published this year by the European Academy of Neurology (EAN) and the European Committee for the Treatment and Research in Multiple Sclerosis (ECTRIMS). These guidelines strive to provide up-to-date, evidence-based recommendations on the treatment of adult patients with clinically isolated syndrome (CIS) or definite MS to guide healthcare professionals in the decision-making process. ${ }^{6}$ Their focus is on DMDs, early treatment in CIS patients, treatment in patients with established disease, monitoring of 


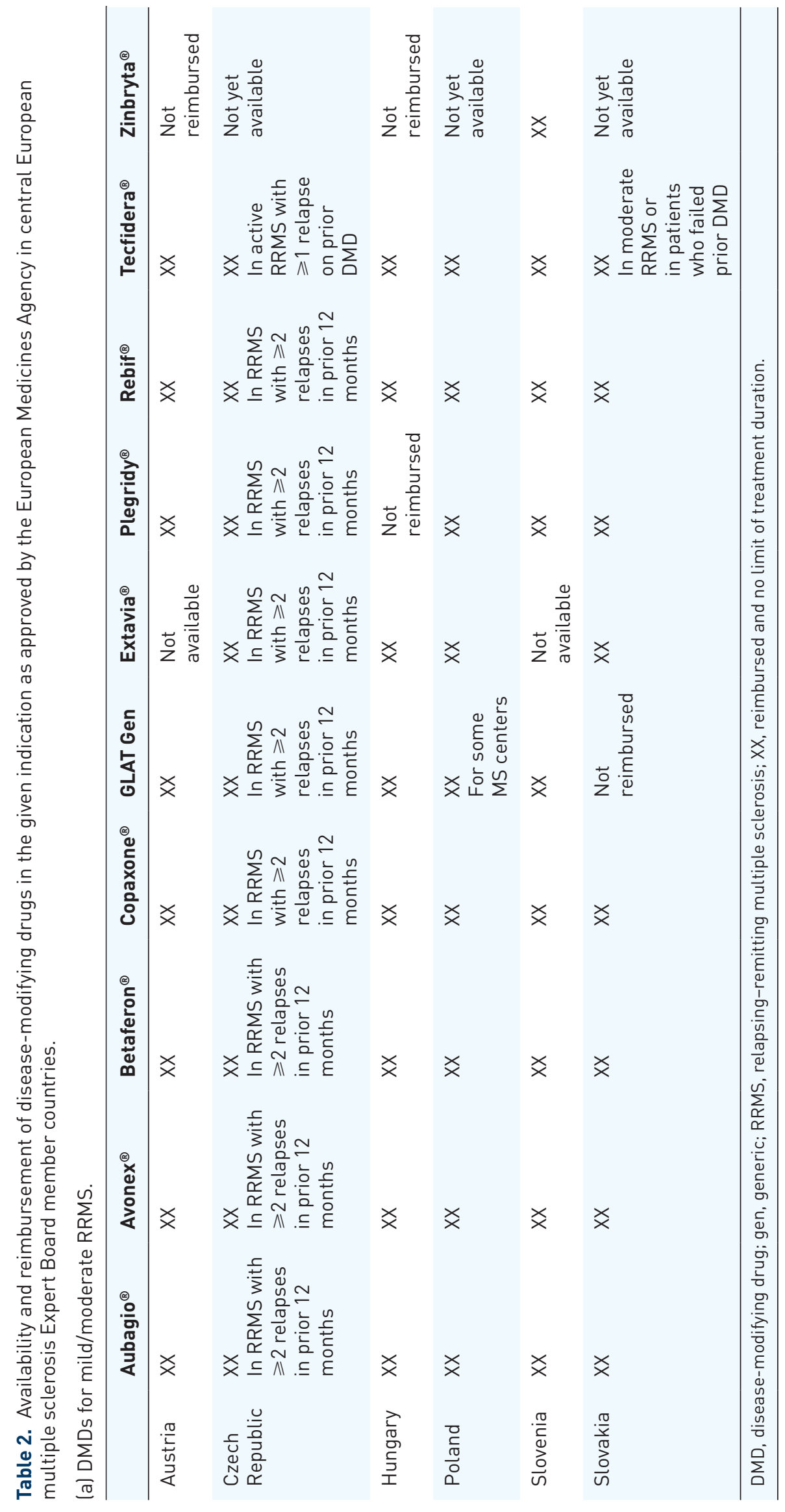




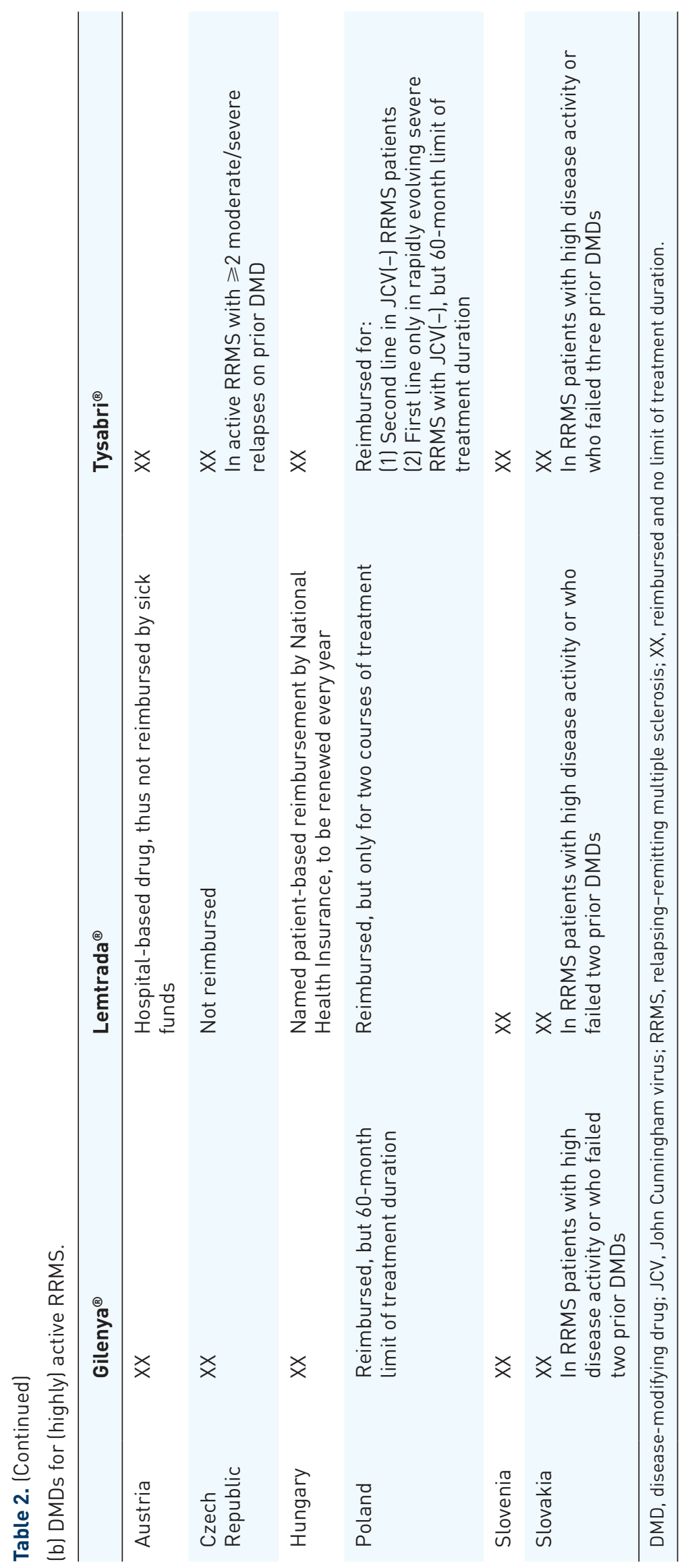


treatment response as well as stopping or switching treatment. These guidelines do not prioritize any of the DMDs, as the available evidence does not inform such a recommendation. Not included are diagnostic guidelines, differential diagnostic guidelines, treatment of acute attacks, symptomatic treatment, and alternative treatments. The recommendations are not intended as a 'cookbook', but can be tailored to local requirements.

It was agreed that it is necessary to challenge the local governments to adhere to the European recommendations instead of finding medical explanations for adjustments that are actually economy driven. Importantly, the new guidelines might provide a basis for argumentation in negotiations with national authorities, thus facilitating improved reimbursement conditions or other amendments. For instance, CIS treatment is still not reimbursed in some European countries even though evidence unequivocally shows that it has beneficial effects.

The expert board cannot stress enough the need for education and specialization. Only qualified experts should treat patients with highly efficacious drugs due to the need for enhanced clinical vigilance and monitoring for treatment effects, including the risk of adverse events. Severe and significant adverse events such as natalizumab-related progressive multifocal leukoencephalopathy (PML) can be overlooked. Educational requirements also extend to neuroradiologists whose obligation it is to recognize the first signs of PML. On the part of the neurologist, clinical judgment can be pivotal here. The guidelines again emphasize that the physician's clinical vigilance and responsibility are at the center of patient management.

From a historical perspective, it is worth mentioning that the ECTRIMS-EAN guideline is not the first of its kind, as there has been international collaboration before. In 2008, the Multiple Sclerosis Therapy Consensus Group (MSTCG) issued therapeutic recommendations on basic and escalating immunomodulatory treatments. ${ }^{7}$

\section{Magnetic resonance imaging as an important interface}

MRI assessment, and thus the collaboration between neurologists and neuroradiologists, has been identified as an interface of growing importance in MS diagnosis and treatment. Issues in this context comprise the lack of standardized protocols for demyelinating disorders, as well as the absence of MS-specific qualification that is frequently observed in the (neuro)radiology community. For reasons of continuity, (neuro)radiologists should interpret a given patient's scans from diagnosis throughout follow up, and the same scanner should be used for each case. On the other hand, neurologists should be familiar with the basics of MRI to be able to communicate appropriately with their imaging partners. Close relationships between neurologists and radiologists are an asset that will facilitate collaboration.

It appears that considerable efforts are required to establish higher levels of quality with regard to the MRI interface. Expert interpretation is currently not available at every center, and consistency is lacking between centers. Rising numbers of scanners in the Eastern countries will increase the need for education of (neuro)radiologists on MS-related quality criteria in the years to come. We endorse the dissemination of the Magnetic Resonance Imaging in MS (MAGNIMS) protocol.8,9 MAGNIMS guidelines need to be discussed and potentially adjusted for central European countries in a collaborative way. Expert MRI reading centers that offer secondary services in the daily routine are a potential solution, but this infrastructural approach must be handled with caution, as existing imaging partners need to be involved.

\section{Contribution of multiple sclerosis registries}

There has been a growing emphasis on the collection and use of real-world data. In spite of several limitations (e.g. collection bias and interpretation bias), MS registries offer advantages such as assessment of drug escalation and medication switches/sequential treatments or the ascertainment of off-label use (e.g. in pediatric MS patients) of approved drugs. This information cannot be obtained by controlled clinical trials. A specific central European registry is currently not realistic, but many centers have started local, national or even international activities. Information on national and international MS registries established in Austria, the Czech Republic, Hungary, Poland, Slovenia, and Slovakia is summarized in Table 3.

Examples of successful registries are the MSBase Registry and the ReMus Czech National Registry. MSBase (www.msbase.org) includes over 40,000 patients worldwide. ${ }^{10,11}$ More than 170 centers are participating in 32 countries. One of the reasons behind the success of this 
Table 3. National and international multiple sclerosis registry participation in central European multiple sclerosis Expert Board member countries.

\begin{tabular}{lll}
\hline & National registry & International registry \\
\hline Austria & $\begin{array}{l}\text { Independent national MS treatment registry since } \\
\text { 2006, participation mandatory for MS centers }\end{array}$ & No \\
$\begin{array}{l}\text { Czech } \\
\text { Republic }\end{array}$ & $\begin{array}{l}\text { Independent national MS registry luses MSBase } \\
\text { data collection tool) }\end{array}$ & MSBase participation \\
Hungary & No, but national MS treatment registry planned & MSBase participation \\
Poland & $\begin{array}{l}\text { National-Health-Fund-based MS treatment } \\
\text { registry, participation mandatory for MS centers }\end{array}$ & No \\
Slovenia & No, but national MS treatment registry planned \\
Slovakia & $\begin{array}{l}\text { Independent national MS registry since 2014, } \\
\text { participation mandatory for neurologists luses } \\
\text { MSBase data collection tool) }\end{array}$ & No \\
\hline MS, multiple sclerosis; & MSBase, international MS registry consortium. & planned
\end{tabular}

registry is the use of the user-friendly iMed (www.imed.org) tool. Also, centers remain the owners of their data. All of the investigators can request access to the dataset for analyses, and are entitled to start substudies. A substantial number of papers have been derived from the MSBase dataset to date. ${ }^{12-37}$

Likewise, the ReMus Czech National Registry (www.nfimpuls.cz), which was established in 2013, has already supplied various publications. The availability of data for negotiations with local authorities was one of the most important objectives for the setup of this registry. It is operated by the endowment fund Impuls, which is the owner of the data, in collaboration with the Czech neuroimmunological society. All of the 15 MS centers in the Czech Republic are contributing to the ReMus Registry. Again, the iMed software is being used to collect the data and report them twice yearly to the technical support organization. The findings are publicly available at the Impuls website (www.multiplesclerosis.cz). Data from almost 9000 patients treated with DMDs have been included up to this point, but enrollment of all MS patients in the Czech Republic is planned for the near future. Comparisons of the quality of care across different regions will become more important, as will assessment of factors such as employment status and time between disease onset and start of DMD treatment.

Finally, a national MS registry was also started in Slovakia in January 2016.

\section{Multiple-sclerosis-related activities in Austria}

The Austrian MS Treatment Registry as well as center-certification and educational processes serve as an example of integration that has continuously been increasing quality standards in the whole country. MS-related activities comprise the MS Treatment Registry, the MS Academy, MS center certification, MS center meetings and quality projects. There is a strong interaction between MS centers, MS societies, health authorities and pharmaceutical companies.

The Austrian MS Treatment Registry has been implemented as a treatment registry with the goals of achieving uniform quality standards of MS treatment documentation in Austria, transparent information for MS centers, alerts to missing control visits and for pharmacovigilance purposes, and conduct of scientific projects. A contract research organization $(\mathrm{CRO})$ has been employed to guarantee maximum data quality by ensuring that the entries are plausible, complete and correct. CRO representatives respond to requests by neurologists at MS centers and contact them to promote high-value data collection. Due to these measures, enormous improvements have been observed over the past 2 years. As entering the data is time consuming for neurologists, a project aims to convince Austrian health insurances to provide specific remuneration for this type of documentation.

MS centers need to qualify for certification by fulfilling a set of requirements. They can be located 
both at hospitals and in the outpatient setting. Currently, there are 100 centers in Austria, but the majority of the Austrian MS patients are being treated at five large centers. Quality projects initiated by the MS network include the Austrian MS Library that was published in 2016. This is a manual containing evidence-based information on all aspects of MS for both patients and physicians.

Together, all of these projects have succeeded in convincing health authorities that the Austrian MS activities are helpful with regard to cost effectiveness. Recommendations have been increasingly accepted, and it is being acknowledged that the registry has led to a gradual gain in quality of care. Fruitful discussion between Austrian health authorities and MS specialists has been promoted on the strength of these efforts. Of course, it would be desirable to implement similar achievements on a large scale in European countries. Therefore, the next central European MS Expert Board meeting will focus exclusively on the harmonization of MS care in the countries represented.

\section{Conclusion}

The participating central European countries have similarities in traditions of medical practice, which can be an opportunity to improve their standards of care of MS patients by learning from each other. On the other hand, there are substantial differences with regard to clinical management of MS, affordability of and access to drugs, and other factors. Pan-European data indicate a west-east gradient for the percentages of patients receiving DMD treatment in general and innovative second-line agents in particular. Insufficient numbers of available neurologists and specifically trained (neuro)radiologists appear to be in widespread shortage. Notably, achieving higher levels of quality is indispensable with respect to cooperation between neurologists and (neuro)radiologists, as MRI diagnostics are a cornerstone of MS management at the time of diagnosis and during follow up.

A common observation in all of the countries is the decline in quality of life with increasing EDSS scores, which is paralleled by steep increases in cost. This underscores the need to implement effective and timely treatment, also with a view to keeping patients in the workforce, which is a significant contributor to the cost-effectiveness of therapies. However, the interest of health authorities in these aspects is often amazingly limited due to the structure of funding systems in various countries that imply the provision of funds by different sources, which are seemingly unconnected. Authorities are encouraged to consider a more holistic view in the interest of patients and health care systems, and to acknowledge overall savings brought about by effective therapies in the long run. The upcoming ECTRIMS-EAN guidelines might contribute to facilitating negotiations with health authorities in the future, as well as pertinent data from MS treatment registries that have already been implemented in several of the participating countries. Existing examples show that combined activities with regard to education, documentation and patient care succeed in improving quality standards over time.

\section{Acknowledgements}

All authors were involved in reviewing the manuscript critically for important intellectual content and provided final approval of the submitted version.

We thank Dr J Moser (Medical Writer, Hoderbergstrasse 21, 3264 Gresten, Austria) for drafting of the manuscript.

Biogen courteously reviewed the draft and provided feedback to the authors. Authors had full editorial control and provided approval to all final content.

\section{Funding}

This advisory expert meeting as well as the drafting of the manuscript by J Moser was funded by Biogen.

\section{Conflict of interest statement}

Monika Adamczyk-Sowa received compensation for travel, advisory boards, speaker honoraria and consultant fees from Bayer, Biogen, Merck, Novartis, Roche, Sanofi Genzyme, and Teva, as well as support for participation in clinical trials in multiple sclerosis sponsored by Bayer and Biogen.

Thomas Berger has participated in the last 12 months in meetings sponsored by, and received honoraria (lectures, advisory boards, consultations) from, pharmaceutical companies marketing treatments for multiple sclerosis: Almirall, Biogen, Celgene, Genzyme, Merck, Novartis, Octapharma, Roche, Sanofi-Aventis and Teva. His institution has received financial support in the last 12 months by unrestricted research grants (Biogen, Novartis, Roche, Sanofi-Aventis and Teva) and for participation in clinical trials in 
multiple sclerosis sponsored by Alexion, Bayer, Biogen, Merck, Novartis, Octapharma, Roche, Sanofi-Aventis and Teva.

Tünde Csépány received speaker honoraria/congress expense compensations from Biogen, Merck Serono, Novartis, Roche, Sanofi Genzyme and Teva.

Eva Havrdová received speaker honoraria and consultant fees from Actelion, Biogen, Celgene, Merck Serono, Novartis, Sanofi Genzyme, Roche and Teva, as well as support for research activities from the Czech Ministry of Education project Progres Q27/LF1.

Saša Šega Jazbec has received speaker honoraria from Biogen, Genzyme, Merck Serono, Novartis.

Dana Horáková was supported by the Czech Ministry of Education project Progres Q27/LF1 and by the Czech Science Foundation GA CR 16-03322S. She also received compensation for travel, speaker honoraria and consultant fees from Bayer, Biogen, Novartis, Merck, Sanofi Genzyme, Roche, and Teva, as well as support for research activities from Biogen.

Tanja Hojs Fabjan received speaker honoraria and consultant fees from Biogen, Genzyme, Lek, Novartis, and for participation in trials in multiple sclerosis sponsored by Bayer and Biogen.

Zsolt Illes has received research support from Biogen, Lundbeckfonden, Merck-Serono, SanofiAventis/Genzyme and travel grants, consultant fees and speaking fees from Bayer Healthcare, Biogen, Merck Serono, Novartis Sanofi-Aventis/ Genzyme and Teva.

Eleonóra Klimová has participated in the last 12 months in meetings sponsored by, and received honoraria (lectures, advisory boards) from, Biogen, Novartis and Teva, and for participation in clinical studies from Biogen and Novartis.

Csilla Rozsa has received personal fees from Biogen, Genzyme, Roche, Novartis and Teva.

Fritz Leutmezer has received speaker honoraria, consultant fees, unrestricted research grants and clinical trial honoraria from Actelion, Biogen, Genzyme, Merck, Novartis, Octapharma, Roche, Sanofi-Aventis and Teva.

Saša Šega Jazbec has received speaker honoraria from Biogen, Genzyme, Merck Serono, Novartis

Jarmila Szilasiová has participated in the last 12 months in meetings sponsored by, and received honoraria (advisory boards, lectures, consultations) from, pharmaceutical companies marketing treatments for multiple sclerosis: Biogen, Genzyme, Merck, Novartis, Roche, Sanofi Aventis, Teva.

Peter Turčáni received honoraria (lectures, advisory boards, consultations) from Biogen, Merck Serono, Novartis, Roche, Sanofi Genzyme and Teva, as well as support for research from Biogen, Novartis and Teva.

László Vécsei has received honoraria (consultations, lectures, chair of sessions) from Biogen, Merck Serono, Novartis, Sanofi-Aventis/Genzyme, Roche and Teva.

\section{References}

1. Kobelt G, Thompson A, Berg J, et al. New insights into the burden and costs of multiple sclerosis in Europe. Mult Scler 2017; 23: 11231136.

2. Karampampa K, Gustavsson A, Miltenburger C, et al. Treatment experience, burden and unmet needs (TRIBUNE) in MS study: results from five European countries. Mult Scler 2012; 18(Suppl. 2): 7-15.

3. Kobelt G, Berg J, Lindgren P, et al. Costs and quality of life of patients with multiple sclerosis in Europe. F Neurol Neurosurg Psychiatry 2006; 77: 918-926.

4. Wilsdon T, Barron A, Mitchell-Heggs A, et al. Access to medicines for multiple sclerosis: challenges and opportunities. CRA Project No. D19380.

5. Flachenecker P, Buckow K, Pugliatti M, et al. Multiple sclerosis registries in Europe: results of a systematic survey. Mult Scler 2014; 20: 1523-1532.

6. Otero-Romero S, Amato MP, Chandraratna D, et al. ECTRIMS-EAN clinical practice guideline on pharmacological management of multiple sclerosis. Mult Scler F 2016; 22 (Suppl. 3): 834.

7. Wiendl H, Toyka KV, Rieckmann P, et al. Basic and escalating immunomodulatory treatments in multiple sclerosis: current therapeutic recommendations. F Neurol 2008; 255: 1449-1463.

8. Rovira À, Wattjes MP, Tintoré M, et al. Evidence-based guidelines: MAGNIMS consensus guidelines on the use of MRI in multiple sclerosis-clinical implementation in the 
diagnostic process. Nat Rev Neurol 2015; 11 : 471-482.

9. Wattjes MP, Rovira À, Miller D, et al. Evidencebased guidelines: MAGNIMS consensus guidelines on the use of MRI in multiple sclerosis: establishing disease prognosis and monitoring patients. Nat Rev Neurol 2015; 11 : 596-606.

10. Butzkueven H, Chapman J, Cristiano E, et al. MSBase: an international, online registry and platform for collaborative outcomes research in multiple sclerosis. Mult Scler 2006; 12 : 769-774.

11. Ziemssen T, Hillert J and Butzkueven H. The importance of collecting structured clinical information on multiple sclerosis. BMC Med 2016; 14: 81 .

12. He A, Spelman T, Jokuabaitis V, et al. Comparison of switch to fingolimod or interferon beta/glatiramer acetate in active multiple sclerosis. FAMA Neurol 2015; 72: 405-413.

13. Hughes SE, Spelman T, Gray OM, et al. Predictors and dynamics of postpartum relapses in women with multiple sclerosis. Mult Scler 2014; 20: 739-746.

14. Hughes SE, Spelman T, Trojano M, et al. The Kurtzke EDSS rank stability increases 4 years after the onset of multiple sclerosis: results from the MSBase Registry. F Neurol Neurosurg Psychiatry 2012; 83: 305-310.

15. Iaffaldano $\mathrm{P}$, Simone $\mathrm{M}$, Lucisano G, et al. Prognostic indicators in pediatric clinically isolated syndrome. Ann Neurol 2017; 81: 729-739.

16. Jokubaitis VG, Spelman T, Kalincik T, et al. Predictors of long-term disability accrual in relapse-onset multiple sclerosis. Ann Neurol 2016; 80: 89-100.

17. Jokubaitis VG, Li V, Kalincik T, et al. Fingolimod after natalizumab and the risk of short-term relapse. Neurology 2014; 82: 1204-1211.

18. Jokubaitis VG, Spelman T, Lechner-Scott J, et al. The Australian Multiple Sclerosis (MS) immunotherapy study: a prospective, multicentre study of drug utilisation using the MSBase platform. PLoS One 2013; 8: e5969.

19. Kalincik T, Brown JWL, Robertson N, et al. Treatment effectiveness of alemtuzumab compared with natalizumab, fingolimod, and interferon beta in relapsing-remitting multiple sclerosis: a cohort study. Lancet Neurol 2017; 16: 271-281.
20. Kalincik T, Kuhle J, Pucci E, et al. Data quality evaluation for observational multiple sclerosis registries. Mult Scler 2017; 23: 647-655.

21. Kalincik T, Jokubaitis V, Izquierdo G, et al. Comparative effectiveness of glatiramer acetate and interferon beta formulations in relapsingremitting multiple sclerosis. Mult Scler 2015; 21: 1159-2271.

22. Kalincik T, Horakova D, Spelman T, et al. Switch to natalizumab versus fingolimod in active relapsing-remitting multiple sclerosis. Ann Neurol 2015; 77: 425-435.

23. Kalincik T, Buzzard K, Jokubaitis V, et al. Risk of relapse phenotype recurrence in multiple sclerosis. Mult Scler 2014; 20: 1511-1522.

24. Kalincik T, Vivek V, Jokubaitis V, et al. Sex as a determinant of relapse incidence and progressive course of multiple sclerosis. Brain 2013; 136: 3609-3617.

25. Kalincik T, Spelman T, Trojano M, et al. Persistence on therapy and propensity matched outcome comparison of two subcutaneous interferon beta 1a dosages for multiple sclerosis. PLoS One 2013; 8: e63480.

26. Kister I, Spelman T, Alroughani R, et al. Discontinuing disease-modifying therapy in MS after a prolonged relapse-free period: a propensity score-matched study. $\mathcal{J}$ Neurol Neurosurg Psychiatry 2016; 87: 1133-1137.

27. Kister I, Chamot E, Cutter G, et al. Increasing age at disability milestones among MS patients in the MSBase Registry. F Neurol Sci 2012; 318 : 94-99.

28. Lechner-Scott J, Spencer B, de Malmanche T, et al. The frequency of CSF oligoclonal banding in multiple sclerosis increases with latitude. Mult Scler 2012; 18: 974-982.

29. Lizak N, Lugaresi A, Alroughani R, et al. Highly active immunomodulatory therapy ameliorates accumulation of disability in moderately advanced and advanced multiple sclerosis. f Neurol Neurosurg Psychiatry 2017; 88: 196-203.

30. Lorscheider J, Buzzard K, Jokubaitis V, et al. Defining secondary progressive multiple sclerosis. Brain 2016; 139: 2395-2405.

31. Rojas JI, Patrucco L, Trojano M, et al. Multiple sclerosis in Latin America: a different disease course severity? A collaborative study from the MSBase Registry. Mult Scler F Exp Transl Clin 2015; 1: 2055217315600193.

32. Spelman T, Meyniel C, Rojas JI, et al. Quantifying risk of early relapse in patients with 
Visit SAGE journals online journals.sagepub.com/ home/tan

@SAGE journals first demyelinating events: prediction in clinical practice. Mult Scler 2017; 23: 1346-1357.

33. Spelman T, Mekhael L, Burke T, et al. Risk of early relapse following the switch from injectables to oral agents for multiple sclerosis. Eur $\mathcal{F}$ Neurol 2016; 23: 729-736.

34. Stewart T, Spelman T, Havrdova E, et al. Contribution of different relapse phenotypes to disability in multiple sclerosis. Mult Scler 2017; 23: $266-276$.

35. Tao C, Simpson S Jr, Van der Mei I, et al. Higher latitude is significantly associated with an earlier age of disease onset in multiple sclerosis. f Neurol Neurosurg Psychiatry 2016; 87: 1343-1349.

36. Trojano M, Lucchese G, Graziano G, et al. Geographical variations in sex ratio trends over time in multiple sclerosis. PLoS One 2012; 7: e48078.

37. Warrender-Sparkes $M$, Spelman $T$, Izquierdo $\mathrm{G}$, et al. The effect of oral immunomodulatory therapy on treatment uptake and persistence in multiple sclerosis. Mult Scler 2016; 22: 520-532. 